\title{
Netzwerken auf Kongressen
}

Die Bewerbungsgespräche sind gelaufen - jetzt kommt die Zeit des Wartens. Bekomme ich die Stelle, die zu mir passt? Und haut das auch zeitlich alles so hin, dass sich alles nahtlos ineinanderfügt?

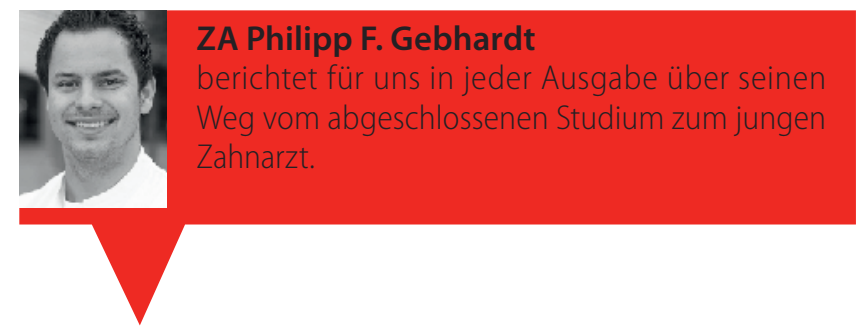

\section{Fortbildung zum Discountpreis}

$\mathrm{W}$ ährend ich auf die Resonanz meiner ersten Bewerbungsunterlagen wartete, versuchte ich die Zeit sinnvoll zu nutzen, jobbte nicht nur, sondern besuchte auch diverse zahnmedizinische Fortbildungen. Ein großer Vorteil war dabei der noch vorhandene Studentenstatus, da Kongressanbieter in diesem Fall meist einen Preisnachlass geben, der nach meinen Erfahrungen sogar bis zu $80 \%$ betragen kann. Und so bekam ich nicht nur weltweit renommierte Referenten zum Discountpreis zu sehen, sondern auch noch die sehr hilfreiche Einsicht, dass mein in Heidelberg erlerntes Wissen absolut zeitgemäß ist.

Interessanterweise habe ich deutschlandweit viele Studenten und Absolventen getroffen, die aufgrund des nicht nur zeitintensiven sondern auch zum Teil stressigen Zahnmedizinstudiums und den damit verbundenen Negativerlebnissen an der Qualität der Ausbildung und ihres Wissensstandes gezweifelt haben. Nicht zuletzt für diese Zweifler kann ein Kongress sowohl eine fachliche Bereicherung sein, als auch das Selbstbewusstsein etwas aufpolieren. Ein weiterer Vorteil ist, dass im Lebenslauf solche extracurriculären Aktivitäten eher positiv ins Gewicht fallen und man sie außerdem auch noch im Folgejahr steuerlich geltend machen kann.

\section{Einstellungsgespräche im Kongresszentrum}

Nun ergab sich der nette Zufall, dass ich in München auf eben solcher Fortbildung den Chef des „Mundwerks" traf. Das „Mundwerk" ist eine der größten kieferorthopädischen Praxen in Berlin und verfügt über eine der genauso begehrten wie seltenen Weiterbildungsberechtigungen. Nach einigen Gesprächen und weiteren Treffen bot er mir die Möglichkeit an, nach dem allgemeinzahnärztlichen Jahr ab dem Januar 2011 bei ihm zu beginnen. Da es nun jedoch schon Dezember 2009 war und keine der von mir angeschriebenen Praxen eine Stelle vor Februar 2010 hatte, befand ich mich in der Zwickmühle. Abgesehen von zwei Regionen in Deutschland ist es nämlich Pflicht, vor Antritt einer Weiterbildungsassistenz in der Kieferorthopädie 12 Monate in Vollanstellung als Zahnarzt gearbeitet zu haben.

Ohne viel Zeit vergehen zu lassen, boten mir meine Eltern an, das Jahr in ihrer Gemeinschaftspraxis zu arbeiten - das war natürlich Glück. Und so stand direkt nach den Examensfeiern ein Umzug aus dem beschaulichen Heidelberg in die Metropole Oschersleben in der Magdeburger Börde an. Super! Noch platt vom Examen, gesättigt von Weihnachten, ohne Urlaub und Silvester im Nacken begann ich also voller Tatendrang mein neues Leben als Zahnarzt.

Berichte ich nun fortan aus Oschersleben? Oder führt mich mein Weg doch noch an andere spannende Stationen? Davon berichte ich nächstes Mal, wenn es heißt „Zurück an die Uni“!

\section{Impressum}

Eigentümer \& Copyright @ Springer-Verlag 2010, Springer Medizin c/o Springer-Verlag GmbH, Tiergartenstr. 17, 69121 Heidelberg, Tel. +49 6221/487-0, www.springer.de. Springer Medizin ist ein Teil der Fachverlagsgruppe Springer Science+Business Media Geschäftsführung Springer Medizin: Harm van Maanen (Executive Vice President), Dr. Dirk Einecke, Ulrich Huber, Stephan Kröck, Dr. Esther Wieland, Matthias Wissel Leitung Zahnmedizin: Dr. Nataša Djordjević (v.i.S.d.P., Tel. -8445, natasa.djordjevic@springer.com)

Redaktion „der junge zahnarzt“: Susanne Redeker (Tel. -8980, susanne.redeker@springer.com), Sabrina Gabriel (Tel.-8170, sabrina.gabriel@springer.com) Layout: Arnulf Illing (Tel. -8952, arnulf.illing@springer.com),

Bildredaktion: Christiane Seufert

Leitung Herstellung: Johannes Buchmann

Zertifizierte Fortbildung: Dr. Paul Herrmann

Gesamtleitung Sales \& Marketing: Stephan Kröck

Anzeigen: Marita Säuberlich (Tel. -8309, Fax -68309, marita.saeuberlich@springer.com) Industriekommunikation: Anja Weisse (Tel. -8739, Fax -68739, anja.weisse@springer.com) Druck: Stürtz GmbH, Würzburg. Printed in Germany

Erscheinungsweise: 4 Ausgaben pro Jahr; Papierausgabe: ISSN 1869-5744, gedruckt auf säurefreiem Papier

Bezugspreise (unverb. Preisempfehlung inkl. 7\% deutscher MwSt. und Versand):
Vorzugspreis für persönliche Abonnenten: EUR 48,-; Einzelheftpreis: EUR 15,-. Das Abonnement kann jederzeit 2 Monate vor Ende des Bezugszeitraumes gekündigt werden. Bestellungen oder Rückfragen nimmt jede Buchhandlung oder der Verlag entgegen. Kontakt: Springer Customer Service Center GmbH, Haberstr. 7, 69126 Heidelberg, Tel. +49 6221/345-4303, Fax-4229, Leserservice@springer.com (Mo.-Fr. 8.00 Uhr bis 20.00 Uhr) Copyright \& allgemeine Hinweise: Die Zeitschrift sowie alle in ihr enthaltenen einzelnen Beiträge und Abbildungen sind urheberrechtlich geschützt. Jede Verwertung, die nicht ausdrücklich vom Urheberrechtsgesetz zugelassen ist, bedarf der vorherigen schriftlichen Zustimmung des Verlags. Das gilt insbesondere für Vervielfältigungen, Bearbeitungen, Übersetzungen, Mikroverfilmungen und die Einspeicherung und Verarbeitung in elektronischen Systemen.

Gezeichnete Artikel geben nicht unbedingt die Meinung der Redaktion wieder. Autoren können unter bestimmten Voraussetzungen an der Ausschüttung der Bibliotheksund Fotokopietantiemen teilnehmen. Einzelheiten bei VG WORT, Abt. Wissenschaft, Goethestr. 49, 80336 München.

Angaben über Dosierungsanweisungen und Applikationsformen sind anhand anderer Literaturstellen oder der Packungsbeilage auf ihre Richtigkeit zu überprüfen. Der Verlag übernimmt keine Gewähr. 